\title{
On Sombor energy of graphs
}

\author{
K. J. Gowtham, Narahari Narasimha Swamy \\ Dept. of Mathematics, University College of Science, Tumkur University, Tumakuru, \\ Karnataka State, Pin 572 103, India \\ gowtham_k_j@yahoo.com,narahari_nittur@yahoo.com
}

DOI 10.17586/2220-8054-2021-12-4-411-417

\begin{abstract}
The concept of Sombor index $S O(G)$ was recently introduced by Gutman in the chemical graph theory. It is a vertex-degree-based topological index and is denoted by $S O(G)$. This paper introduces a new matrix for a graph $G$, called the Sombor matrix, and defines a new variant of graph energy called Sombor energy $\operatorname{ES}(G)$ of a graph $G$. The striking feature of this new matrix is that it is related to well-known degree-based topological indices called forgotten indices. When $\operatorname{ES}(G)$ values of some molecules containing hetero atoms are correlated with their total $\pi$ electron energy, we got a good correlation with the correlation coefficient $r=0.976$. Further, we found some bounds and characterizations on the largest eigenvalue of $S(G)$ and Sombor energy of graphs.
\end{abstract}

Keywords: Sombor index, Sombor energy, forgotten index.

Received: 30 May 2021

Revised: 1 July 2021

Final revision: 20 July 2021

\section{Introduction}

Spectral graph theory plays an important role in analyzing the matrices of graphs with the help of matrix theory and linear algebra. Now, spectral graph theory has attracted the attention of both pure and applied mathematicians whose benefit lies far from the spectral graph theory, which may be surprised because graph energy is a special kind of matrix norm. They will then recognize that the concept of graph energy (under different names) is encountered in several seemingly unrelated areas of their own expertise. The eigenvalues are closely related to almost all major invariants of a graph, linking one extremal property to another, they play a central role in the fundamental understanding of graphs [1]. In 1978, Gutman related the Graph energy and total $\pi$-electron energy in a molecular graph; it was defined as, the sum of absolute values of the eigenvalues of the associated adjacency matrix of a graph $G$ [2]. Later, many matrices were defined based on distance and adjacency among the vertices, degree of the vertices involved in forming the graph structure like: Zagreb matrix [3], Randić matrix [4], distance matrix [5], Seidel matrix [6], Laplacian matrix [7], Seidel Laplacian matrix [8], signless Laplacian matrix [9], Seidel signless Laplacian matrix [10], degree sum matrix [11], etc.

Topological indices are mainly categorized into two types: namely degree-based indices and distance-based indices. Some of the well-known degree-based indices are first Zagreb index, second Zagreb index, forgotten index, hyper Zagreb index, Randic index, harmonic index, geometric-arithmetic index, redefined third Zagreb index, inverse sum index, etc. The details on degree-based topological indices we refer to [12-14]. Recently, Gutman et al. [15] defined new degree-based indices, called the Sombor index, which is one of the trending areas in the present graphtheoretical research. The details of this new index we refer to [16-25]. In [26], the chemical applicability of Sombor indices had been studied. The wide application of Sombor indices motivated us to define the Sombor matrix and Sombor energy of the graph.

\section{Preliminaries}

In this paper, we considered simple, finite, undirected, and connected graphs. A graph $G$ involves a vertex set $V=V(G)=\left\{v_{1}, v_{2}, \ldots, v_{n}\right\}$ and $E=E(G)$ as its edge set. If two vertices have a common edge, then they are known as adjacent vertices. Likewise, if two edges have a common end vertex, then it is called an adjacent edge. The number of edges incident to a vertex $v$ is called the degree of that vertex $v$ and it is denoted by, $d_{v}$

The first and second Zagreb indices of a graph $G$ [2] is defined as,

$$
M_{1}(G)=\sum_{u v \in E(G)}\left(d_{u}+d_{v}\right)=\sum_{u \in V(G)} d_{u}^{2} \text { and } M_{2}(G)=\sum_{u v \in E(G)}\left(d_{u} d_{v}\right)
$$

respectively. 
Followed by the above definition, Furtula and Gutman introduced the forgotten topological index [27], defined as:

$$
F(G)=\sum_{u v \in E(G)}\left(d_{u}^{2}+d_{v}^{2}\right)=\sum_{u \in V(G)} d_{u}^{3} .
$$

In [15] Gutman defined new degree based topological index called Sombor index, denoted by $S O(G)$ and defined as:

$$
S O(G)=\sum_{u v \in E(G)} \sqrt{\left(d_{u}^{2}+d_{v}^{2}\right)},
$$

In the next section, we introduce a new matrix for a graph $G$ and a new graph invariant based on this matrix.

\section{Sombor matrix and Sombor energy of graph}

Definition 3.1. The Sombor matrix of a graph $G$ with a vertex set $V=V(G)=\left\{v_{1}, v_{2}, v_{3}, \ldots, v_{n}\right\}$ and edge set $E=E(G)$ is defined as $S(G)=\left(s_{i j}\right)_{n \times n}$, where:

$$
s_{i j}= \begin{cases}\sqrt{d_{u}^{2}+d_{v}^{2}}, & u v \in E(G) \\ 0, & \text { otherwise }\end{cases}
$$

where $d_{u}$ denotes the degree of the vertex $u$.

The Sombor polynomial of a graph $G$ is defined as:

$$
P_{S(G)}(\lambda)=|\lambda I-S(G)|,
$$

where $I$ is an $n \times n$ unit matrix.

Since $S(G)$ is real symmetric matrix, all roots of $P_{S(G)}(\lambda)=0$ are real. Hence, they can be arranged as $\lambda_{1} \geq \lambda_{2} \geq \lambda_{3} \cdots \geq \lambda_{n}$. The Sombor energy of graph $G$ is given by

$$
E S(G)=\sum_{i=1}^{n}\left|\lambda_{i}\right| .
$$

\section{Chemical applicability of $E S(G)$}

The development of Huckel molecular orbital theory is mainly concentrated on conjugated, all carbon compounds. The range of those compounds can be studied if hetero atoms are considered. This can be done by comparing energy values for hetero compounds. To this end, we need to adjust Coulomb $(\alpha)$ and resonance integral $(\beta)$ values for hetero atoms using the relations:

$$
\alpha^{\prime}=\alpha+h \beta \quad \text { and } \quad \beta^{\prime}=k \beta,
$$

where $h$ and $k$ are correction values which are different and depending on what atom is in conjugation. So, we can take more than one value for $\alpha$ for a hetero atom but depends on the number of electrons hetero atom donates to $\pi$-system.

Consider, the secular matrix of the compound urea:

$$
\left[\begin{array}{cccc}
\alpha-E & 1.131 \beta & \beta & 0 \\
1.131 \beta & \alpha+1.5 \beta-E & 0 & 0 \\
\beta & 0 & \alpha+\beta-E & 0 \\
0 & 0 & 0 & \alpha+1.5 \beta-E
\end{array}\right]
$$

By substituting appropriate values of $\alpha$ and $\beta$ for urea in the above matrix and expanding the secular determinant, $\pi$-electron energy for urea can be calculated $[28,29]$ we found the close resemblance between secular matrix of hetro molecule and Sombor matrix $S(G)$ of corresponding molecular graph $G$. Further, we calculated $E S(G)$ with dataset of total $\pi$-electron energy values of hetero atoms which are found in [30]. From Fig. 1 we found that $E S(G)$ has good correlation hetero atoms, as mentioned in Fig. 2, with correlation coefficient $r=0.976$ and $r^{2}$ (adjusted) $=0.952$. 


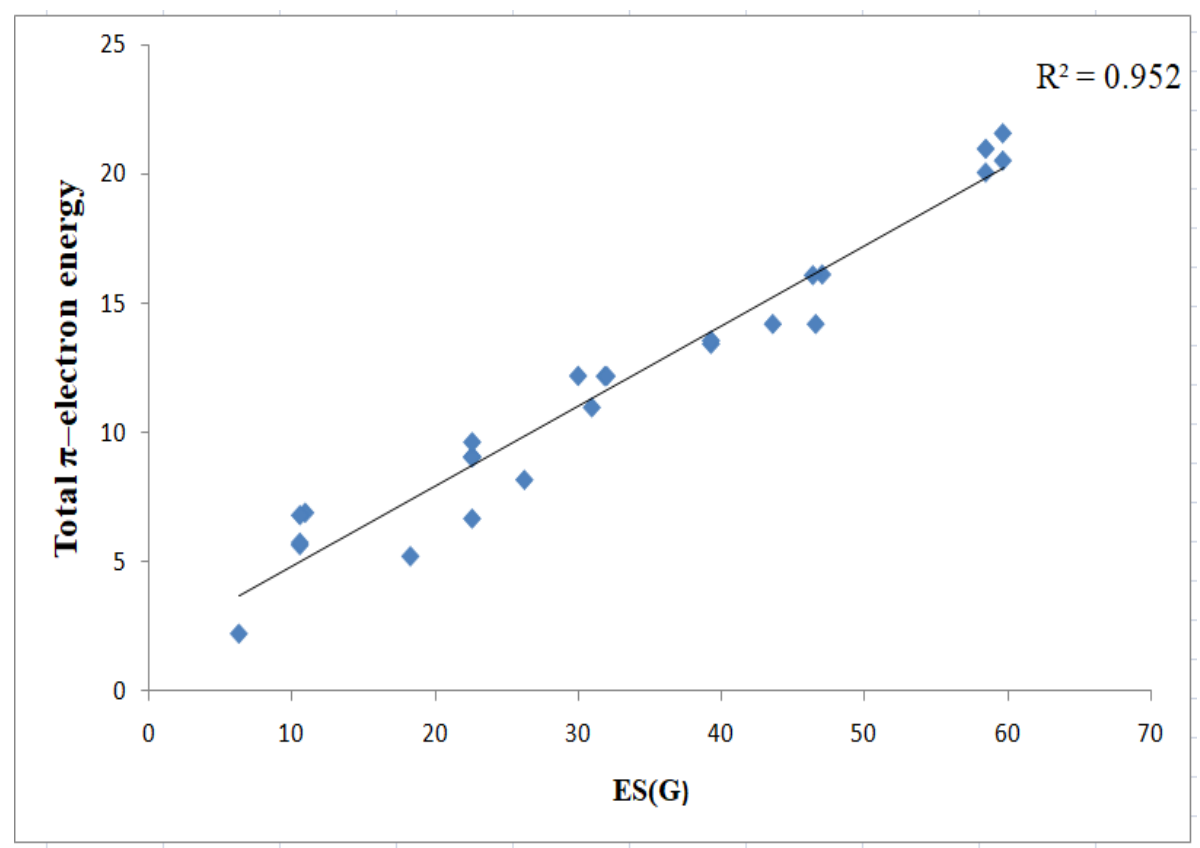

FIG. 1. Correlation of $E S(G)$ with the total $\pi$-electron energy of molecules containing hetero atoms

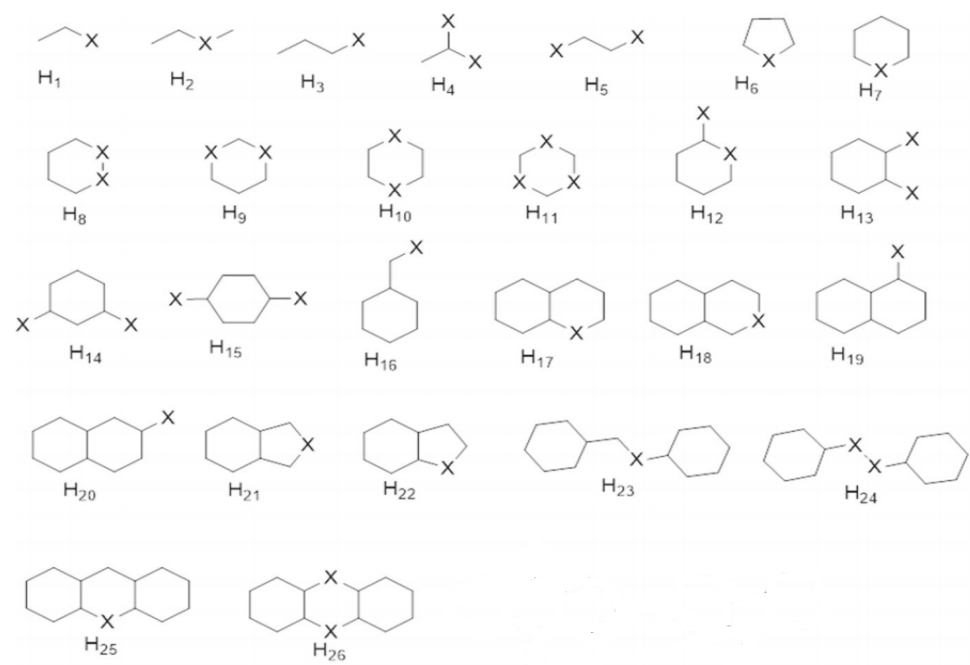

FIG. 2. Molecules containing hetero atoms. $\left(\mathrm{H}_{1}=\right.$ Venyl chloride like systems, $\mathrm{H}_{2}=$ Butadiene perturbed at $\mathrm{C}_{2}, \mathrm{H}_{3}=$ Acrolein like systems, $\mathrm{H}_{4}=1$,1-Dichloro-ethylene like systems, $\mathrm{H}_{5}=\mathrm{Glyoxal}$ like and 1,2-Dichloro-ethylene like systems, $\mathrm{H}_{6}=$ Pyrrole like systems, $\mathrm{H}_{7}=$ Pyridine like systems, $\mathrm{H}_{8}=$ Pyridazine like systems, $\mathrm{H}_{9}=$ Pyrimidine like systems, $\mathrm{H}_{10}=$ Pyrazine like systems, $\mathrm{H}_{11}=\mathrm{S}$ Triazene like systems, $\mathrm{H}_{12}=$ Aniline like systems, $\mathrm{H}_{13}=\mathrm{O}$-Phenylene-diamine like systems, $\mathrm{H}_{14}=\mathrm{m}$ Phenylene-diamine like systems, $\mathrm{H}_{15}=\mathrm{p}$-Phenylene-diamine like systems, $\mathrm{H}_{16}=$ Benzaldehyde like systems, $\mathrm{H}_{17}=$ Quinoline like systems, $\mathrm{H}_{18}=$ Iso-quinoline like systems, $\mathrm{H}_{19}=1$-Naphthalein like systems, $\mathrm{H}_{20}=2-\mathrm{Naphthalein} \mathrm{like} \mathrm{systems,} \mathrm{H}_{21}=$ Iso-indole like systems, $\mathrm{H}_{22}=$ Indole like systems, $\mathrm{H}_{23}=$ Benzylidine-aniline-like systems, $\mathrm{H}_{24}=$ Azobenzene like systems, $\mathrm{H}_{25}=$ Acridine like systems, $\mathrm{H}_{26}=$ Phenazine like systems) 


\section{Some result on Sombor matrix}

Theorem 5.1. Let $G$ be graph with vertex set $V(G)$ and edge set $E(G)$ with the Sombor matrix $S(G)$. If

$$
P_{S(G)}=c_{0} \lambda^{n}+c_{1} \lambda^{n-1}+c_{2} \lambda^{n-2}+\cdots+c_{n}
$$

is the characteristic polynomial of $S(G)$, then

i. $c_{2}=-F(G)$,

ii. $c_{3}=-2 \sum_{\Delta} \prod_{u v \in E(\Delta)} \sqrt{d_{u}^{2}+d_{v}^{2}}$,

where $\Delta$ is a triangle in the graph $G$.

Proof. $\quad$ i. From the definition of $P_{S(G)}$, we have:

$$
c_{2}=\sum_{1 \leq i<j \leq n}\left|\begin{array}{cc}
0 & s_{i j} \\
s_{j i} & 0
\end{array}\right|=-\sum_{1 \leq i<j \leq n} s_{i j}^{2}=\sum_{u v \in E(G)} d_{u}^{2}+d_{v}^{2}=-F(G) .
$$

ii. From the definition of $P_{S(G)}$, we have:

$$
c_{3}=-\sum_{1 \leq i<j \leq n}\left|\begin{array}{ccc}
0 & s_{i j} & s_{i k} \\
s_{j i} & 0 & s_{j k} \\
s_{k i} & s_{k j} & 0
\end{array}\right|=-\sum_{1 \leq i<j<k \leq n} s_{i j} s_{j k} s_{k i}=-2 \sum_{\Delta} \prod_{u v \in E(\Delta)} \sqrt{d_{u}^{2}+d_{v}^{2}} .
$$

Lemma 5.2. [Newton's identity] Given an $n \times n$ matrix $A$, let $P(\lambda)=c_{0} \lambda_{n}+c_{1} \lambda_{n-1}+c_{2} \lambda_{n-2}+\cdots+c_{n}$ be the characteristic polynomial of $A$, then the coefficient $c_{3}=\frac{1}{6}\left[-(\operatorname{Tr}(A))^{3}+3 \operatorname{Tr}(A) \operatorname{Tr}\left(A^{2}\right)-2 \operatorname{Tr}\left(A^{3}\right)\right]$.

Theorem 5.3. Let $S(G)=\left(s_{i j}\right)_{n \times n}$ be the Sombor matrix of a graph $G$ and be its $\lambda_{1} \geq \lambda_{2} \geq \cdots \geq \lambda_{n}$ eigenvalues, then:
i. $\sum_{i=1}^{n} \lambda_{i}^{2}=2 F(G)$,
ii. $\sum_{i=1}^{n} \lambda_{i}^{3}=3 \sum_{\Delta} \prod_{u v \in E(\Delta)} \sqrt{d_{u}^{2}+d_{v}^{2}}$

where $\Delta$ is a triangle in the graph $G$

Proof.

i. $\sum_{i=1}^{n} \lambda_{i}^{2}=\operatorname{Tr}\left(S(G)^{2}\right)=\sum_{i=1}^{n} \sum_{j=1}^{n} s_{i j} s_{j i}=\sum_{i=1}^{n} s_{i i}^{2}+\sum_{i \neq j} s_{i j} s_{j i}=2 \sum_{i<j} s_{i j}^{2}=2 \sum_{u v \in E(G)} d_{u}^{2}+d_{v}^{2}=2 F(G)$.

ii. We know that, $\operatorname{Tr}\left(S(G)^{3}\right)=\sum_{i=1}^{n} \lambda_{i}^{3}$. The result follows by equating the coefficient $c_{3}$ values given in Theorem 5.1, Lemma 5.2 and using the facts $\operatorname{Tr}(S(G))=0$ and $\operatorname{Tr}\left(S(G)^{2}\right)=2 F(G)$. We get the required result.

\section{Bounds for the largest eigenvalue}

Theorem 6.1. If $G$ is any graph with $n$ verties with $S(G)=\left(s_{i j}\right)_{n \times n}$ being its Sombor matrix and $\lambda_{1} \geq \lambda_{2} \geq \cdots \geq$ $\lambda_{n}$ are its eigenvalues, then

$$
\lambda_{1} \leq \sqrt{2(n-1) F(G)} .
$$

Proof. Taking $a_{i}=\lambda_{i}$ and $b_{i}=1$ for $i=1,2,3, \ldots, n$ in Cauchy-Schwarz inequality we get:

$$
\left(\sum_{i=2}^{n} \lambda_{i}\right)^{2} \leq(n-1) \sum_{i=2}^{n} \lambda_{i}^{2}
$$

On solving we get:

$$
\begin{aligned}
\left(-\lambda_{1}\right)^{2} & \leq(n-1)\left(2 F(G)-\lambda_{1}^{2}\right) \\
\Longrightarrow \quad \lambda_{1} & \leq \sqrt{2(n-1) F(G)} .
\end{aligned}
$$




\section{Bounds for Sombor energy}

Now we obtain some bounds on the Sombor energy of graphs. To this end, we make use of the following classical inequalities

Lemma 7.1. [Diaz-Metcalf Inequality] Let $\left(a_{1}, a_{2}, \ldots, a_{n}\right)$ and $\left(b_{1}, b_{2}, \ldots, b_{n}\right)$ be positive real numbers, satisfying the condition $r a_{i} \leq b_{i} \leq R a_{i}$ for $1 \leq i \leq n$. Then:

$$
\sum_{i=1}^{n} b_{i}^{2}+r R \sum_{i=1}^{n} a_{i}^{2} \leq(r+R) \sum_{i=1}^{n} a_{i} b_{i}
$$

Equality holds if and only if $b_{i}=R a_{i}$ or $b_{i}=r a_{i}$ for $1 \leq i \leq n$.

Lemma 7.2. Let $a_{1}, a_{2}, \ldots, a_{n}$ be non-negative real numbers, then:

$$
n\left(\frac{1}{n} \sum_{i=1}^{n} a_{i}-\left(\prod_{i=1}^{n} a_{i}\right)^{1 / n}\right) \leq n \sum_{i=1}^{n} a_{i}-\left(\sum_{i=1}^{n} \sqrt{a_{i}}\right)^{2} \leq n(n-1)\left(\frac{1}{n} \sum_{i=1}^{n} a_{i}-\left(\prod_{i=1}^{n} a_{i}\right)^{1 / n}\right) .
$$

\subsection{Lower bounds for the Sombor energy}

Theorem 7.3. Let $G$ be any graph with $n$ verties and let $P$ be the absolute value of the determinant of Sombor matrix $S(G)$, then:

$$
\sqrt{2 F(G)+n(n-1) P^{2 / n}} \leq E S(G) .
$$

Proof.

$$
[E S(G)]^{2}=\left(\sum_{i=1}^{n}\left|\lambda_{i}\right|\right)^{2}=\sum_{i=1}^{n}\left|\lambda_{i}\right|^{2}+\sum_{i \neq j}\left|\lambda_{i}\right|\left|\lambda_{j}\right|=2 F(G)+\sum_{i \neq j}\left|\lambda_{i}\right|\left|\lambda_{j}\right| .
$$

Clearly we have:

$$
\frac{1}{n(n-1)} \sum_{i \neq j}\left|\lambda_{i}\right|\left|\lambda_{j}\right| \geq \prod_{i \neq j}\left(\left|\lambda_{i}\right|\left|\lambda_{j}\right|\right)^{1 /(n(n-1))}=\left|\prod_{i=1}^{n} \lambda_{i}\right|^{2 / n}=P \frac{2}{n} \Longrightarrow \sum_{i \neq j}\left|\lambda_{i}\right|\left|\lambda_{j}\right| \geq n(n-1) P^{2 / n}
$$

therefore

$$
\sqrt{2 F(G)+n(n-1) P^{2 / n}} \leq E S(G) .
$$

Theorem 7.4. Let $G$ be a graph with $n$ verties. Then

$$
\frac{2 F(G)+n\left|\lambda_{1}\right|\left|\lambda_{n}\right|}{\left|\lambda_{n}\right|+\left|\lambda_{1}\right|} \leq E S(G),
$$

where, $\left|\lambda_{1}\right|$ and $\left|\lambda_{n}\right|$ are maximum and minimum of the absolute value of eigenvalues of $S(G)$. Equality will be attained if and only if for each $1 \leq i \leq n$, either $\left|\lambda_{i}\right|=\left|\lambda_{1}\right|$ or $\left|\lambda_{i}\right|=\left|\lambda_{n}\right|$.

Proof. substituting $b_{i}=\left|\lambda_{i}\right|, a_{i}=1, r=\left|\lambda_{n}\right|$ and $R=\left|\lambda_{1}\right|$ in lemma 7.1, we have

$$
\begin{aligned}
& \sum_{i=1}^{n}\left|\lambda_{i}\right|^{2}+\left|\lambda_{n}\right|\left|\lambda_{1}\right| \sum_{i=1}^{n} 1 \leq\left(\left|\lambda_{1}\right|+\left|\lambda_{1}\right|\right) E S(G) \\
& \Longrightarrow \frac{2 F(G)+n\left|\lambda_{1}\right|\left|\lambda_{n}\right|}{\left|\lambda_{1}\right|+\left|\lambda_{n}\right|} \leq \operatorname{ES}(G)
\end{aligned}
$$




\subsection{Upper bound for Sombor index}

Theorem 7.5. If $G$ is a graph with $n$ verties, then

$$
E S(G) \leq \sqrt{n F(G)}
$$

Proof. Put $a_{i}=1$ and $b_{i}=\left|\lambda_{i}\right|$ in Cauchy-Schwarz inequality, we get

$$
[E S(G)]^{2} \leq n\left(\sum_{i=1}^{n}\left|\lambda_{i}\right|\right)^{2}=n F(G)
$$

Simplifying the above equation we get the required result.

Theorem 7.6. If $G$ is a graph with $n$ verties, then:

$$
E S(G) \leq \sqrt{n\left\{2 F(G)+\left[\operatorname{Det}\left(N(G)^{2}\right)\right]^{1 / n}\right\}-2 F(G)},
$$

where $|\operatorname{Det}(N(G))|$ is absolute value of the determinant of Sombor matrix $S(G)$.

Proof. Substituting $a_{i}=\lambda_{i}^{2}$ for $i=1,2, \ldots, n$ in lemma 7.2, we have:

$$
n\left(\frac{1}{n} \sum_{i=1}^{n} \lambda_{i}^{2}-\left(\prod_{i=1}^{n} \lambda_{i}^{2}\right)^{1 / n}\right) \leq n \sum_{i=1}^{n} \lambda_{i}^{2}-\left(\sum_{i=1}^{n} \lambda_{i}\right)^{2} .
$$

Using results in theorem 5.1, we get:

$$
n\left(\frac{1}{n} 2 F(G)-|\operatorname{Det}(S(G))|^{1 / n}\right) \leq n 2 F(G)-(E S(G))^{2} .
$$

On simplifying above equation we arrive the required result.

\section{Conclusion}

Recently, Gutman introduced a new vertex-degree-based topological index, called the Sombor index $S O(G)$ in chemical graph theory. In this paper, we have introduced a new matrix for a graph $G$, called the Sombor matrix, and defined a new variant of graph energy called the Sombor energy $E S(G)$ of a graph $G$. The striking feature of this new matrix is that it is related to the well-known degree-based topological indices called forgotten indices. When $E S(G)$ values of some molecules containing hetero atoms are correlated with their total $\pi$-electron energy, we obtained a good correlation with the correlation coefficient $r=0.952$. Further, bounds ( lower and upper) and characterizations on the largest eigenvalue of $S(G)$ and Sombor energy of graphs have been studied.

\section{References}

[1] Boregowda H.S., Jummannaver R.B. Neighbors degree sum energy of graphs. J. Appl. Math. Comput., 2021, https://doi.org/10.1007/s12190020-01480-y.

[2] Gutman I., Trinajstić N. Graph theory and molecular orbitals. Total $\pi$-electron energy of alternant hydrocarbons. Chem. Phys. Lett., 1972, 17, P. 535-538.

[3] Jafari Rad N., Jahanbani A., Gutman I. Zagreb Energy and Zagreb Estrada Index of Graphs. MATCH Commun. Math. Comput. Chem., 2018, 79, P. 371-386.

[4] Rajendra P., Randić. Color Energy of a Graph. Int. Journal of Computer Applications, 2017, 171, P. 1-5.

[5] Aouchiche M., Hansen P. Distance spectra of graphs: a survey. Linear Algebra Appl., 2014, 458, P. $301-386$.

[6] Brouwer A., Haemers W. Spectra of Graphs, Springer, Berlin, 2012.

[7] Mohar B. The Laplacian spectrum of graphs. In: Alavi, Y., Chartrand, G., Ollermann, O.R., Schwenk, A.J. (eds.) Graph Theory, Combinatorics and Applications, Wiley, New-York, 1991, 2, P. 871-898.

[8] Ramane H.S., Jummannaver R.B., Gutman I. Seidel Laplacian energy of graphs. Int. J. Appl. Graph. Theory, 2017,2 , P. 74-82.

[9] Cvetković D., Rowlinson P., Simić S.K. Eigenvalue bounds for the signless Laplacian. Publ. Inst. Math. (Beograd), 2007,81, P. $11-27$.

[10] Ramane H.S., Gutman I., Patil J.B., Jummannaver R.B. Seidel signless Laplacian energy of graphs. Math. Interdiscip. Res., 2017, 2, P. 181192.

[11] Ramane H.S., Revankar D.S., Patil J.B. Bounds for the degree sum eigenvalues and degree sum energy of a graph. Int. J. Pure Appl. Math. Sci., 2013, 6, P. 161-167.

[12] Das K.C., Trinajstić N. Comparison between first geometric-arithmetic index and atom-bond connectivity index. Chem. Phys. Lett., 2010, 497, P. 149-151. 
[13] Graovac A., Gutman I., Trinajstić N. Total $\pi$-Electron Energy. In: Toplogical Approach to the Chemistry of Conjugated Molecules, 1977, 4, P. $48-78$.

[14] Gutman I. Degree-based topological indices. Croat. Chem. Acta, 2013, 86, P. 351-361.

[15] Gutman I. Geometric approach to degree-based topological indices: Sombor indices. MATCH Commun. Math. Comput. Chem., 2021, 86, P. 11-16.

[16] Das K.C., Cevik A.S., Cangul I.N., Shang Y. On Sombor Index. Symmetry, 2021, 13, 140.

[17] Cruz R., Gutman I., Rada J. Sombor index of chemical graphs. Applied Mathematics and Computation, Elsevier, 2021, 399, 126018.

[18] Deng H., Tang Z., Wu R. Molecular trees with extremal values of Sombor indices. Int. J. Quantum. Chem., 2021, 121 (11), e26622.

[19] Gutman I. Some basic properties of Sombor indices. Open J. Discret. Appl. Math., 2021, 4, P. 1-3.

[20] Horoldagva B., Xu C. On Sombor index of graphs. MATCH Commun. Math. Comput. Chem., 2021, 86, P. 703-713.

[21] Kulli V.R., Gutman I. Computation of Sombor Indices of Certain Networks. Int. Journal of Applied Chemistry, 2021, 8, P. 1-5.

[22] Liu H., You L., Tang Z., Liu J.B. On the reduced Sombor index and its applications. MATCH Commun. Math. Comput. Chem., 2021, 86, P. 729-753.

[23] Milovanović I., Milovanović E., Matejić M. On some mathematical properties of Sombor indices. Bull. Int. Math. Virtual Inst., 2021, 11, P. 341-353.

[24] Réti T., Doslić T., Ali A. On the Sombor index of graphs. Contrib. Math., 2021, 3, P. 11-18.

[25] Wang Zh., Mao Y., Li Y., Furtula B. On relations between Sombor and other degree-based indices. J. Appl. Math. Comput., 2021, doi:10.1007/s12190-021-01516-x.

[26] Redžepović I. Chemical applicability of Sombor indices. J. Serb. Chem. Soc., 2021, 86 (5), P. 445-457.

[27] Furtula B., Gutman I. A forgotten topological index. J. Math. Chem., 2015, 53, P. 1184-1190.

[28] Ramachandran K.I., Deepa G., Namboori K. Computational Chemistry and Molecular Modelling: Principles and Applications. Springer, Berlin, 2008.

[29] Yates K. Huckel Molecular Orbital Theory. Academic Press, New York, 1978.

[30] Coulson C.A., Streitwieser J. Dictionary of $\pi$-Electron Calculations. Freeman, San Francisco, 1965. 